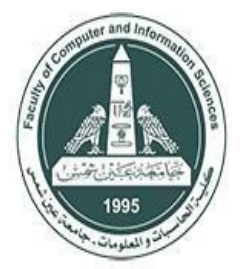

International Journal of Intelligent Computing and Information Sciences

https://ijicis.journals.ekb.eg/

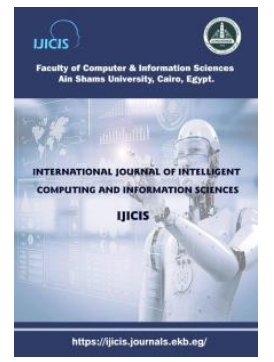

\title{
MOBILE CROWDSENSING FRAMEWORK FOR ROAD SURFACE QUALITY DETECTION
}

\author{
Karim Emara ${ }^{\text {a }}$ \\ karim.emara@cis.asu.edu.eg \\ Aya El-kady* a \\ aya.elkady@cis.asu.edu.eg \\ Eman Shaaban ${ }^{\text {a }}$ \\ eman.shaaban@cis.asu.edu.eg \\ Mohamed Hamdy ElEliemy ${ }^{\text {a,b }}$ \\ m.hamdy@cis.asu.edu.eg \\ a: Faculty of Computer and Information Sciences, Ain Shams University, Cairo, Egypt \\ b: Faculty of Computing and Information Technology, King Abdulaziz University, 21589 Jeddah, Saudi Arabia
}

Received 2021- 8-24; Revised 2021-9-30; Accepted 2021-10-7

Abstract - Smartphones became ubiquitous and are used by so many people, at least to know the driving directions to their destination. Smartphones come with rich embedded sensors (e.g., GPS, accelerometer, and camera) as well as built-in radios (e.g., Bluetooth, Wi-Fi, and Cellular), which both enable users to gather data and distribute it among people at any time or location. These features have come up with the mobile crowdsensing (MCS) development which can be used in a wide range of applications. In this paper, we introduce a complete mobile crowdsensing framework for road surface condition detection. Various modules have been addressed such as task management, data fusion, reputation scoring, incentive awarding, security, and privacy, as well as discussing popular techniques and algorithms utilized in the proposed MCS framework modules. A prototype of the crowd sensing application is designed which is related to our framework. The proposed framework considers the data quality and trustiness between the users and the server as well.

Keywords: Mobile crowdsensing, road anomalies, smartphone sensors, framework.

\section{Introduction}

Smartphone sensing as well as social networking approaches have a significant impact on people's lives and led to a better standard of living [1]. The smartphone's wide usage has come up with both the mobile crowdsensing and crowd computing concepts. Mobile sensing can be very helpful in sharing daily information and spread knowledge to improve lifestyle. Mobile crowdsensing development can be used in a

* Corresponding author: Aya El-kady

Faculty of Computer and Information Sciences, Ain Shams University, Cairo, Egypt

E-mail address: aya.elkady@cis.asu.edu.eg 
wide range of applications usually categorized into three types: infrastructure (such as detecting road anomalies), environmental (such as monitoring noise), and social (such as tracking the movement and exercises of participants). Mobile crowdsensing (MCS) relies on collecting data from various users using sensors in their smartphones to improve the quality and reliability of data. This paper focuses on the utilization of mobile crowd-sensing in the detection of road surface conditions. Poor road conditions such as bumps, potholes, and broken pavement can cause traffic congestion, vehicle damages, and accidents. Many road anomalies do not have visible labels or aren't visible at all especially at night and in bad weather conditions. Most of the existing methods for monitoring road conditions like radar, cameras, and ultra-sonic detectors are expensive and cannot be adopted to cover the whole road network, but they are deployed or used in a small portion of the system for main roads. Industrial-level accelerometer sensors can be used in detecting road conditions, but they will not be an efficient way as the number of vehicles that will have this sensor installed will not be large enough to cover the whole network. Nowadays, smartphones and tablets are used everywhere and carried by most people.

In this paper, an MCS framework is proposed which describes the modules and mechanisms needed to build a reliable trusted system for detecting the road surface conditions. The introduced framework consists of several interacted modules proposing various quality-aware mechanisms including user management, task assignment, data acquisition, privacy-preserving data fusion, classification, reputation score and incentive calculation, and visualization. First, the server keeps a record of monitored roads and regularly assigns sensing tasks to be executed by the collectors. In data acquisition, the smartphone sensors are employed to return reading samples which are preprocessed before being collected by the aggregator (server). Several data fusion and privacy algorithms are discussed to ensure the reliability and security of user's data shared within the system. Next, a machine learning classification technique is utilized to identify if a set of sensor readings represents a road anomaly or not. However, to build such a classifier, collected datasets should be labeled to be used in the training phase. A clustering technique, called DBSCAN [2], is utilized to accomplish this data labeling automatically. Several reputation scoring mechanisms are explained and compared regarding the data features. The presented algorithms are the robust average [3] and the Gompertz function[3-5], where each of them is responsible for calculating a score that indicates the trustworthiness of users. Finally, a rewarding system is adopted to encourage users to actively participate in the system and gather more data. Therefore, our contributions can be summarized as follows:

- Introduce a complete mobile crowdsensing framework for road surface condition detection.

- Survey and discuss popular techniques and algorithms utilized in the proposed MCS framework modules.

- Design a prototype of a crowd sensing application that implements the proposed framework.

Next, we will discuss related work. In Section 3, the framework is illustrated showing its main modules. We show conclusions and future work 4 in

\section{Related Work}

In this section a short review about the literature on proposed mobile crowd sensing frameworks. Guo et. Al [4] have characterized the main attributes of a mobile-crowded system (MCS), including data collection, data mining, and mentioned how to deal with Low-Quality Data. They proposed a generic MCS framework consisting of five layers: crowdsensing, data transmission, data collection, crowd data processing, and applications. They highlighted the strengths and weaknesses between human and machine intelligence and discussed the vision of mixing them in an MCS system. Authors of [6] proposed a robust framework for road anomaly detection that relies on wide experiments of different car models equipped with multiple 
smart devices and integrated positioning units. The sensed data from these activities were used to create data sets with different types of events. A de-noising technique is applied to the data to reduce the noise effect. Also, feature extraction techniques are adopted to efficiently characterize the impact of road irregularities on the collected data. These data sets were used in training a multilevel support vector machine (SVM) classifier which can effectively detect multiple anomalies with varying levels of danger with an average TPR performance of $90 \%$. Authors of [7] proposed an MCS framework for collecting data that reflects different phenomenon such as road traffic and climate data. A smartphone application is implemented for data collection and a cloud is utilized to acts as a service platform to help in integrating and analyzing the data to define certain phenomena in certain roads Data analysis was performed in a compressed form to decrease the capacity of the storage. A prototype mobile application has been released, used for collecting crowdsourced data. Feature extraction is applied on the axes of the accelerometer and gyroscope readings using a sliding window of 2-second duration. The features being extracted are root-mean-square, standard deviation, correlation, high-frequency energy, low-frequency energy and mean speed. An SVM is used in the classification of the road conditions depending on the one-versus-one (OVO) technique since the SVM cannot cope with multi-class classification but binary ones. In the testing process, the voting strategy "MaxWins" is used to generate the output. "FixMyStreet" [8], is a website that permits compatriots to report problems in the road like potholes and other road anomalies by spotting the exact location of the problem on a map. Also, the website is used to determine damaged, dirty, ripped items, which need repairing or cleaning. The reports are sent to the concerned council to fix it. Users can discuss road issues with other people on the website and then either send them to the council or take action by themselves. Citizens reporting a problem are reached by the system within four weeks to follow up the issues if they have been fixed or not. Authors of [9] showed a taxonomy for various MCS applications. They stated that the paradigm of crowd participation is divided into two modes: direct and word of mouth (WOM) modes. They present a generic framework that consists of various modules and illustrate the typical workflow of MCS applications. Three stakeholders are concerned in the system: task publishers, crowd workers, and crowdsourcing platform, and the role of each of them is discussed. It has pointed out the properties and challenges of integrated MCS systems, also pointed out their disadvantages.

\section{Framework}

Figure 1 shows the proposed framework, which consists of the following modules:

\section{A. User Management module}

The user management module is responsible for registering users whether data collectors or consumers and keeping track of their user information. First, users must register to the system choosing whether to be a consumer or a collector. A consumer is a typical user who takes benefit of the system features. A consumer can obtain the road condition of its trajectory by simply communicating with the server and providing its trajectory, then a map pinned with road structure and condition will be viewed in the smartphone app. A collector is by default a consumer who can take benefit of the system features but in addition, can accept data collection tasks assigned by the server. A data collection task is mainly a request for road condition detection in a specific area and is governed by some criteria such as energy level, reputation score, and sensor presence. 
The user management module will keep track of all registered users, their roles, and profiles. In the collector's profile, an incentive mechanism is enabled to maintain the rewarding points obtained for the accomplished tasks. Also, a reputation score is calculated based on the quality of provided data.

\section{B. Task assignment module}

The task assignment module requests tasks from collectors to acquire the required data for a specific road or area. Collectors can review these tasks and accept applicable ones according to their locations and preference. An approval from the module is required to select the most suitable collectors to do the task. The approval is governed by checking the following criteria:

- Sensor presence: The collector's device should be equipped with a list of sensors required for the requested task. These sensors include Accelerometer, Gyroscope, Gravity, and Magnetometer.

- Energy level: the collector's device should have a sufficient energy level to ensure the completeness of the collected data [10].

- Reputation score: The task is assigned only to collectors with an acceptable reputation score to ensure the trustworthiness of the collected data. The method of calculating this score will be described later in the reputation module.

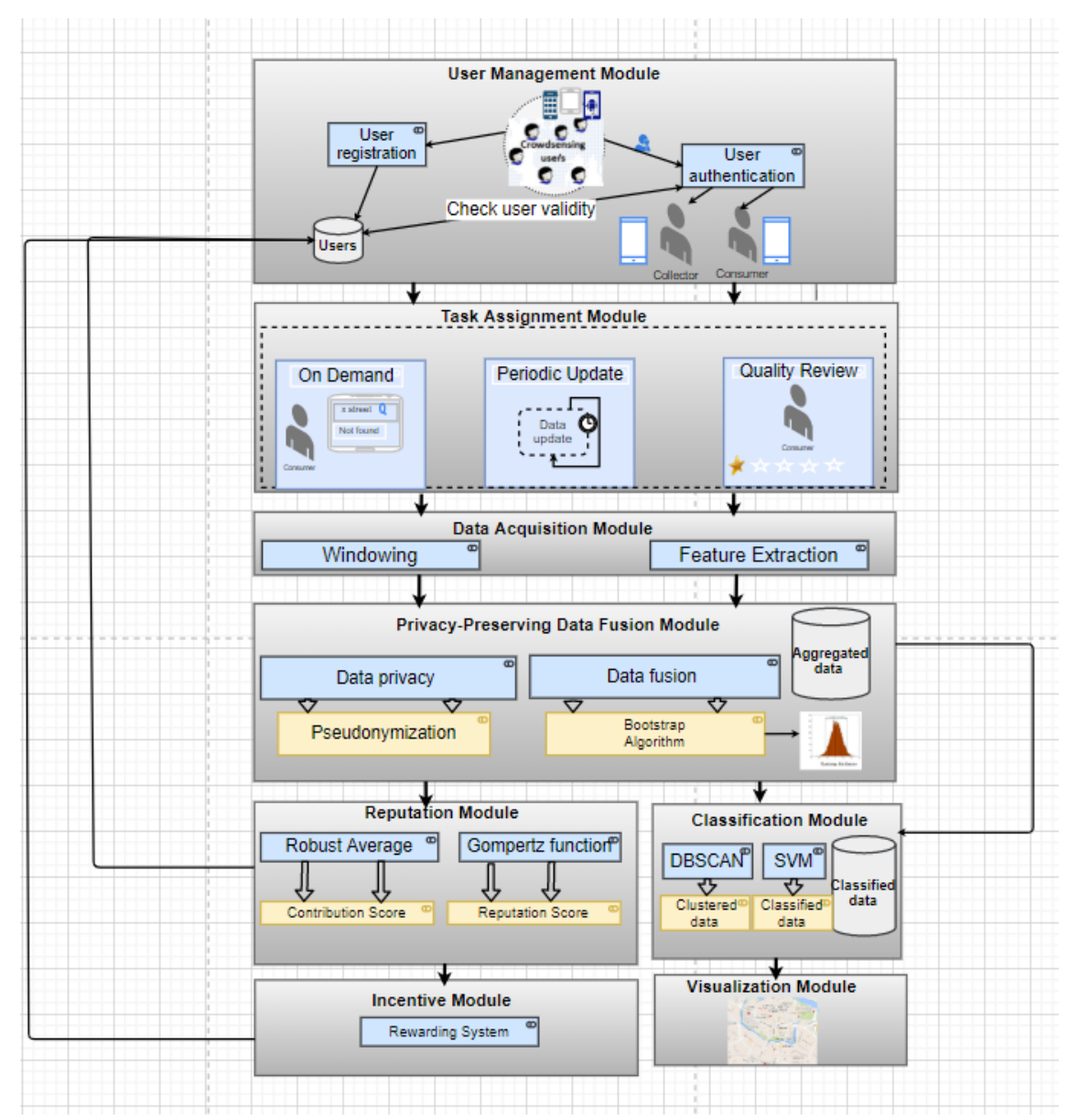


In general, tasks are generated based on consumer's direct requests, their feedback reviews, and periodic updates for the previously collected road conditions. Therefore, tasks can be categorized into three types: on-demand, quality review, and periodic update.

\section{On-Demand:}

This task type is generated when a consumer requests the condition of a certain road or area. If the requested road is not evaluated before and no information about it is found in the database, then the task assignment module transfers this request to a task that should be assigned to one of the qualified collectors.

\section{Quality Review:}

This task type is generated in response to users' negative reviews for received road conditions. The data should be recollected if the user feedback is repeatedly negative for the given road, which indicates data inaccuracy. The task assignment module establishes a new task concerning this specific area. The newly collected data is compared with the stored data to determine if the stored data was corrupted, or it was a fake review from the consumer to confuse the system.

\section{Periodic Update:}

Since the condition of the road network continuously changes, the collected data should be periodically updated to ensure the quality and integrity of the provided service. Two main reasons support the need for periodic updates triggered by the server. Firstly, due to the rapid expansion of the road network, there will always be areas that are not covered. Therefore, the task assignment module should periodically select and prioritize uncovered areas and generate the corresponding tasks proactively for the data collectors to be ready for the consumer requests. Secondly, the road network is regularly changed due to possible anomalies occur or repairs done by the authority. Thus, the road condition data of the covered areas should be periodically re-checked in case that the server did not receive any updates from the collectors or reviews from consumers about this data. In this case, the stored data becomes outdated, and the task assignment module should schedule a task to collect information concerning this area.

\section{Data acquisition module}

Collecting data for the assigned tasks is accomplished by the data acquisition module at the collector side. The data acquisition module focuses on accurately collecting data using smartphone sensors and improving the data quality before being processed. The sensor readings are recorded from the considered sensors which are typically the accelerometer, magnetometer, gyroscope, and gravity sensors as well as the GPS [11]. The accelerometer measures the relative change in velocity including gravity. Therefore, a linear acceleration sensor that provides the acceleration excluding the force of gravity is usually used. The magnetometer and gravity sensors are used in a reorientation process, where the smartphone's axes must be aligned with the axes of the vehicle. This makes the data collection more flexible for the collector because the smartphone can be placed at any position inside the vehicle. All sensors run at the highest available frequency to attain a sampling rate of $10 \mathrm{~Hz}$ which is accurate enough to detect a road anomaly. The data 
are then written as records stored at the internal storage of the collector's smartphone. The data record consists of the following fields:

1. Timestamp in milliseconds

2. Raw linear acceleration in $\mathrm{x}, \mathrm{y}$ and $\mathrm{z}$ coordinates

3. Readings of the magnetometer, gravity and gyroscope sensors

4. Transformed linear acceleration.

5. Speed calculated by measuring the delta in distance and time between two consecutive GPS locations

6. The longitude and latitude acquired from the GPS.

Before data analysis, individual records are grouped into time windows [12] of one-second duration to extract features for each window. The considered features are the mean and the standard deviation of the sensor readings (field 4) in the three coordinates along with the average speed and GPS longitude and latitude. The extracted features are returned from the collector to the server to be analyzed with the features obtained from other collectors in the same area.

\section{Privacy-preserving data fusion module}

\section{Data Fusion}

Since a data collection task is assigned to several collectors in the same area, several extracted features are received to the server. These data records should be fused to obtain more consistent and accurate information than that provided by any individual collector. Data fusion is the process of integrating multiple data sources to produce more useful information. Data fusion mechanism is not a simple task as data are gathered by different crowd-sensing collectors with different vehicles and smartphones and data may be collected over a period in which the road surface may be changed. A distribution method called "bootstrap" [13] is considered to get statistics of the aggregation result of the multiple collectors. The GPS coordinates are extracted from the data and converted to the corresponding military grid reference system (MGRS) grid index. A grid square of size $10 \mathrm{~m}$ is chosen. The grid square consists of four digits, the longitude used to determine the first and third digits, the second and fourth ones are determined by the latitude.

For each grid, the data is gathered through different time epochs. For each epoch t, (i.e., the time frame from which data of all participating users is collected), a sample is obtained from all participants which is then entered into a resampling process where several resamples are obtained from it without the need of forcing the users to collect data several times to obtain more samples. The mean value is calculated for each new sample and plotted into a certain block. This mean value is updated with each time epoch by being averaged with its corresponding value computed at the previous epoch. The results are then forming the output bootstrap distribution which is used for obtaining the confidence interval. Finally, we consider only this interval to working with while ignoring the other. This leads to an increase in the quality and confidence of data.

\section{Data Privacy}

Since the quality and reliability of data are improved when gathered from various collectors "crowd sensors", data fusion techniques should consider the factor of security as well. Collectors send their sensed data attached with their exact location and time, and this can be repeated every time the participant accepts a 
task. This can lead to exposing their private information to the service providers in a non-convenient way. Service providers can know about users' places of interest and the time of being there. Because of these privacy issues, participants may hesitate or even retreat to send their information to the system. Assurance of their data security and privacy is a must to encourage users to participate in the assigned tasks and send their sensed data with their obfuscated personal information without any anxiety.

Authors in [14] proposed an anonymous data aggregation protocol that utilizes a source anonymity algorithm. It considers the existence of a non-trusted mediator that aggregates user's data without being able to recognize the source of these data in a crowdsensing scenario. A user sends time-series data to the mediator over a secured channel after performing its assigned sensing task. Bitwise-XOR homomorphic encryption is applied where the data of all users are encrypted using their own private keys then the aggregator can compute a bitwise XOR of all user's data just by calculating the bitwise XOR of ciphertexts of all users. In [15], homomorphic encryption is also applied but users add some noise to the data before being encrypted. In [16], users cannot determine how much noise to add but the noise distribution is customized by the platform.

Since the sensed data required for road condition application should be accurate, obfuscation techniques will affect data quality and therefore reduce the system accuracy in the detection of road anomalies. In addition, anonymized homomorphic encryption techniques prevent the server from accounting for the collectors' participation in the assigned tasks and make the reputation score calculation and rewarding mechanism adoption harder. Therefore, a more convenient data privacy mechanism that can be applied in the proposed framework is pseudonymization. Pseudonymization is a privacy-preserving technique where the personally identifiable information fields within a data record are replaced by one or more artificial identifiers called pseudonyms. By using pseudonyms, the data privacy becomes more preserved while keeping the data suitable for data analysis and processing. In the proposed framework, collectors interact with a pseudonym provider to obtain a pool of pseudonyms using their system credentials obtained during the user registration phase. A pseudonym provider is a trusted third party who keeps the mapping of a user identity to their pseudonyms secret. While accomplishing their assigned tasks, collectors send the needed sensor data identified by one of their pseudonyms to the data fusion module. The reputation score and rewarding points are then calculated for the pseudonyms and returned to the registered users with the support of the pseudonym provider.

\section{E. Classification module}

Sensed data and extracted features aggregated by the data fusion module should be processed and classified to identify the road surface quality. In the classification module, as shown in Figure 2, machine learning techniques are utilized in 1) labeling road surface datasets automatically and then 2) training a classification model that can evaluate the road surface quality. Automatic labeling is crucial to generalize the adopted methodology when dealing with very large datasets since manual labeling is impractical in that case.

Authors in [17] introduced a generic methodology for automatically labeling the collected dataset whether each window of sensor readings reflect a road anomaly or normal road. This methodology evaluated KMeans and density-based clustering (DBSCAN) [2] algorithms and found that DBSCAN achieves a better accuracy than K-Means with minimum accuracy of $96 \%$ when compared with the manually labeled datasets. The DBSCAN algorithm achieves a higher accuracy because it is effective in finding nonlinear shapes of clusters based on the density. It is used in dividing the feature vectors extracted from the dataset windows into different clusters. DBSCAN does not require a priori specification of the number of clusters and can identify noisy data and outliers. Automatic labeling is crucial to generalize the adopted 
methodology when dealing with very large datasets. Once datasets are labeled, the classification model is trained to be used in predicting the road surface quality of unseen sensor readings. The labeled datasets are used to train a Support Vector Machine (SVM) classification model. Then, the resultant classifier is used to classify new sensor readings that are captured by the collector to determine if it represents a normal road or a road anomaly.

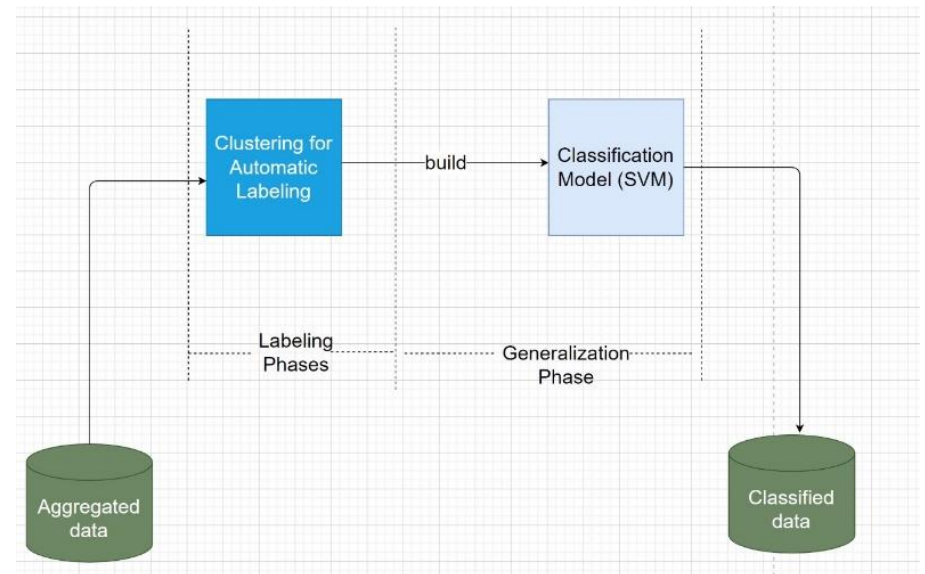

Figure 2: Block diagram for the classification module

\section{F. Reputation module}

The aim of adopting a reputation system in the framework is to build a trust relationship between users and ensure data reliability. Previous studies have addressed this system through various approaches.

Authors in [18] proposed a trust framework for detecting untrustworthy participant contributions depending on their attitude. The proposed method consists of two parts of assessments: subjective and objective parts. Where the participant has to send two parts of data about a certain event, the first is the subjective assessment which is his personal report or review, and the other is the objective one, which is the raw sensor data. Therefore, these two types of values require different types of users' participation; participatory which is related to the subjective event values and the other is opportunistic which is related to the objective event values where involvement of the user is minimal. In order to detect fake users, it deduces the characteristics of a user's assessment disposition from their subjective assessments of the events. Kolmogorov-Smirnov (KS) test is used to compare each participant with other ones separately and find similar users depending on their attitudes. Authors of [19] proposed a trust mechanism called E-R to maintain and create trust relationships between mobile device users in an MCS platform. It depends on two factors of trust: an experience that indicates the relationship between users and reputation which reflects the behavior of the user in the community. An indirect interaction occurs between the data provider and service consumer, where the data provider uploads data to the MCS platform to be forwarded to the service consumer after calculating the quality of data value. Based on this quality, the "interaction value attribute" between the two users is calculated. After every interaction between trustor and trustee, the experience should be handled to correctly indicate the relationship between the two users. The reputation of each user is computed based on previous experiences between users accordingly. Finally, experience and reputation are fused to compute a trust a relationship between the users.

A system is proposed by Huang et al. [3] for the assessment of the trustworthiness of participants' contributions in mobile crowd sensing systems. They introduced a trust framework that consists of a watchdog module and a reputation module. In the watchdog module, a cooperative rating is assigned to each 
user's device to detect the probability of the device's cooperation. The watchdog module produces this rating by executing an outlier detection algorithm based on majority voting or robust average algorithms. The ratings of the contributions are then normalized to be input to the reputation module that computes a reputation score based on "Gompertz function".

For our framework, we followed the system proposed in [3] which considers the two scoring modules: the watchdog and reputation modules to distinguish between the cooperative users and the non-cooperative ones.

\section{Watchdog module:}

The watchdog module aims to determine the probability of the device's cooperation by utilizing outlier detection algorithms such as density-based, robust average and majority voting algorithms. The robust average algorithm is adopted in our framework, as follows. First, GPS coordinates are placed on a grid and for each grid cell, a contribution score for each user in each time epoch is calculated. The collected data is divided into time epochs $\mathrm{K}$ of 10 seconds duration each. The considered data is the extracted features for each $1 \mathrm{~s}$ window, as mentioned earlier in the data acquisition module. However, the standard deviation in the $\mathrm{z}$-axis is only used, as it is shown that this feature alone provides similar road surface classification accuracy [20]. In each time epoch, there is a vector that consists of 10 values of the standard deviation in the z-direction. Data from user $i$ in a certain time epoch $\mathrm{K}$ can be represented by a vector. $X_{(i, k)}=\left[x_{i, t}, \ldots, x_{i, t+T-1}\right]$, where $\mathrm{T}=10 \mathrm{~s}$ and with $\mathrm{t}=(\mathrm{k}-1) * \mathrm{~T}+1$, in that epoch. A robust average value is calculated for each data value in each time epoch $\mathrm{k}$, as follows:

$$
r_{t}=\sum_{i=1}^{n} p_{i, k} x_{i, t}(k-1) \times T<t \leq K \times T
$$

where $\mathrm{p}_{\mathrm{i}, \mathrm{k}}$ is the contribution score for user $\mathrm{i}$ in each time epoch $\mathrm{k}$ and its value $>=0$ as defined in Eq. (2). It applies to all standard deviation values. A device can be said to be cooperative if its $p_{i, k}>=1 / n$, where $n$ is the number of contributors in this epoch.

$$
p_{i, k}=\frac{\frac{1}{\sum_{i=1}^{n} \sum_{t=1}^{T}\left(x_{i, t}-r_{t}\right)^{2}}+\varepsilon}{\sum_{j=1}^{n} \frac{1}{\frac{\sum_{t=1}^{T}\left(x_{j, t}-r_{t}\right)^{2}}{\sum_{i=1}^{n} \sum t=1^{T}\left(x_{i, t}-r_{t}\right)^{2}}}}+\varepsilon
$$

It's clear from the two equations that the $\boldsymbol{P}_{\boldsymbol{i}, \boldsymbol{k}}$ and $\boldsymbol{r}_{\boldsymbol{t}}$ values are iterative. $\boldsymbol{p}_{\boldsymbol{i}, \boldsymbol{k}}$ is initialized by $1 / \mathrm{n}$ then updated at each iteration till reaching the convergence using "Gompertz function [5] [3]. Finally, $\boldsymbol{R}_{\boldsymbol{i}, \boldsymbol{k}}$ shown in Eq. (3), is used to specify the trusted users from others. The reputation score of each collector is then stored in the users' database.

$$
R_{i, k}\left(p^{\prime}{ }_{i, k}\right)=a e^{b e^{c p \prime_{i, k}}}
$$


Where $p^{\prime}{ }_{i, k}$ is the normalized contribution score after adding to it an aging weight in order to reduce the impact of past data.

\section{G. Incentive module}

To encourage users to participate in the system, an incentive mechanism should be adopted to compensate them with rewards for their time and battery consumption, also for their honesty and care to deliver real data about the required task. Incentive mechanisms are being introduced in several works. An application in [21] is considered as a game, and it rewards the citizens according to their activity. It designs a reward system so that more points are gained based on fixing the issues not only reporting them. Also, points are gained by users through voting or reporting abuse users. Not only points are given when sending the data but also when providing the feedback needed to rate each user in the system.

Authors in [15] introduced a Reliable Hybrid Incentive Mechanism. It decides that the sensing users can obtain three kinds of incentives: reward payment, reputation integral incentive, and service quality incentive. This is to ensure the reliability of the data as well as the long-term participation of a sufficient number of sensing users. Another incentive mechanism" TruCentive" is proposed in [22] that fits into its system which is "Crowdsourced Parking Availability Systems". In this system, contributors provide the information of when and where a parking spot is available or soon-to-be-available and called this information "PA information". The function of "TruCentive" is to supply a system for contributors and consumers to "trade" PA information. System credits are used as the incentive for each PA information is transferred between contributors and consumers. In order to encourage users to share PA information, two types of rewards are being paid by the system: a static reward and a bonus reward. The static reward is afforded immediately after a PA message is accepted by TruCentive. The bonus reward is afforded right after the parking is confirmed a success, that is, the consumer has really benefited from the PA message and succeeded in finding a parking spot. For the proposed framework, we consider a rewarding system where points are gained by the contributors who accomplish a sensing task completely with an acceptable reputation to the system. More points are gained if the collector accepts the critical tasks "unknown areas". Collectors then take benefits from these points by getting offers in some transport applications like Uber, Careem ....etc.

\section{H. Visualization module}

Our application prototype is described in Figure 3. The registered users log in to the system and then they are redirected to the page related to their profile, whether a consumer or collector. In the case of a collector, the assigned tasks are listed and give the option to accept one or more tasks. If the collector acceptance is approved by the system, then the sensing phase starts, and the user's smartphone begins to collect the data and send them to the server. The points rewarded for the collector always appear at the top of its page. In the case of a consumer, the service page appears where the user can enter his destination where its route is calculated and showing the road anomalies as pins on the map. Once the user reaches his destination, the system will ask for his evaluation of the service. 

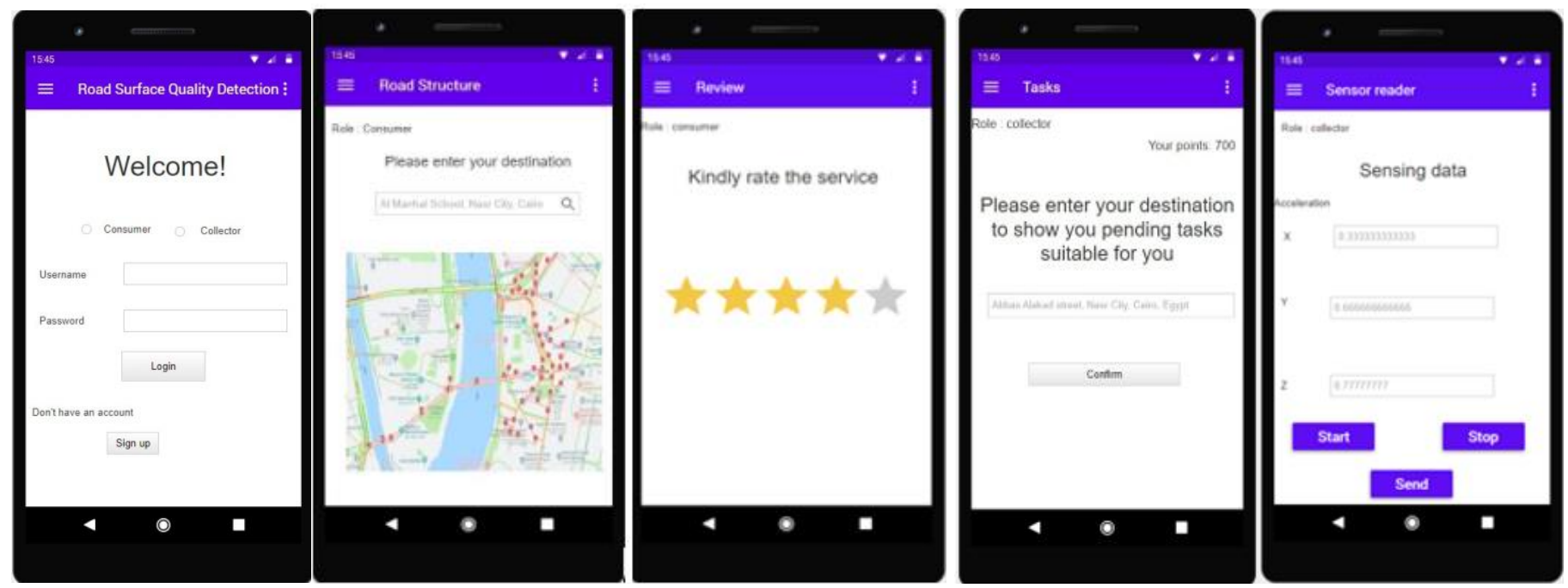

Figure 3:Application prototype

\section{Conclusion}

The increase in smartphone usage has encouraged the utilization of the embedded sensors to detect road surface quality. In this paper, a framework is proposed to illustrate the different modules needed to design a trusted and efficient system for road surface condition detection. We suggested different algorithms and techniques in each module. Different issues have been addressed such as task management, data fusion, reputation scoring, incentive awarding, security, and privacy. The illustration of these modules can pave the way for academic researchers, or developers to implement a realistic MCS tool. Our future work is to implement the proposed framework on the cloud and a mobile platform and evaluate it using datasets collected while driving on different roads.

\section{References}

1. alhumayani, m., M. Monir, and r. ismail, machine and deep learning approaches for human activity recognition. International Journal of Intelligent Computing and Information Sciences, 2021: p. 1-9.

2. Campello, R.J., D. Moulavi, and J. Sander. Density-based clustering based on hierarchical density estimates. in Pacific-Asia conference on knowledge discovery and data mining. 2013. Springer.

3. Huang, K.L., S.S. Kanhere, and W. Hu, On the need for a reputation system in mobile phone based sensing. Ad Hoc Networks, 2014. 12: p. 130-149.

4. Guo, B., Z. Yu, X. Zhou, and D. Zhang. From participatory sensing to mobile crowd sensing. in 2014 IEEE International Conference on Pervasive Computing and Communication Workshops (PERCOM WORKSHOPS). 2014. IEEE.

5. Tjørve, K.M. and E. Tjørve, The use of Gompertz models in growth analyses, and new Gompertzmodel approach: An addition to the Unified-Richards family. PloS one, 2017. 12(6): p. e0178691. 
6. El-Wakeel, A.S., J. Li, A. Noureldin, H.S. Hassanein, and N. Zorba, Towards a practical crowdsensing system for road surface conditions monitoring. IEEE Internet of Things Journal, 2018. 5(6): p. 4672-4685.

7. Aihara, K., H. Imura, B. Piao, A. Takasu, and Y. Tanaka, Mobile crowdsensing to collect road conditions and events, in Smart Sensors at the IoT Frontier. 2017, Springer. p. 271-297.

8. King, S.F. and P. Brown. Fix my street or else: using the internet to voice local public service concerns. in Proceedings of the 1st international conference on Theory and practice of electronic governance. 2007.

9. Wang, Y., X. Jia, Q. Jin, and J. Ma, Mobile crowdsourcing: framework, challenges, and solutions. Concurrency and Computation: Practice and experience, 2017. 29(3): p. e3789.

10. Liu, C.H., Z. Chen, and Y. Zhan, Energy-efficient distributed mobile crowd sensing: A deep learning approach. IEEE Journal on Selected Areas in Communications, 2019. 37(6): p. 1262-1276.

11. Kok, M., J.D. Hol, and T.B. Schön, Using inertial sensors for position and orientation estimation. arXiv preprint arXiv:1704.06053, 2017.

12. sayed, d.a., S. rady, and M. Aref, SCLUSTREAM: AN EFFICIENT ALGORITHM FOR TRACKING CLUSTERS OVER SLIDING WINDOW IN BIG DATA STREAMING. International Journal of Intelligent Computing and Information Sciences, 2019. 19(2): p. 1-19.

13. Freschi, V., S. Delpriori, E. Lattanzi, and A. Bogliolo, Bootstrap based uncertainty propagation for data quality estimation in crowdsensing systems. Ieee access, 2017. 5: p. 1146-1155.

14. Zhang, Y., Q. Chen, and S. Zhong, Privacy-preserving data aggregation in mobile phone sensing. IEEE Transactions on Information Forensics and Security, 2016. 11(5): p. 980-992.

15. Xiong, J., R. Ma, L. Chen, Y. Tian, L. Lin, and B. Jin, Achieving incentive, security, and scalable privacy protection in mobile crowdsensing services. Wireless Communications and Mobile Computing, 2018. 2018.

16. Yang, L., M. Zhang, S. He, M. Li, and J. Zhang. Crowd-empowered privacy-preserving data aggregation for mobile crowdsensing. in Proceedings of the Eighteenth ACM International Symposium on Mobile Ad Hoc Networking and Computing. 2018.

17. El-Kady, A., K. Emara, M.H. ElEliemy, and E. Shaaban. Road Surface Quality Detection using Smartphone Sensors: Egyptian Roads Case Study. in 2019 Ninth International Conference on Intelligent Computing and Information Systems (ICICIS). 2019. IEEE.

18. Zupančič, E. and B. Žalik, Data trustworthiness evaluation in mobile crowdsensing systems with users' trust dispositions' consideration. Sensors, 2019. 19(6): p. 1326.

19. Truong, N.B., G.M. Lee, T.-W. Um, and M. Mackay, Trust evaluation mechanism for user recruitment in mobile crowd-sensing in the Internet of Things. IEEE Transactions on Information Forensics and Security, 2019. 14(10): p. 2705-2719.

20. Bhoraskar, R., N. Vankadhara, B. Raman, and P. Kulkarni. Wolverine: Traffic and road condition estimation using smartphone sensors. in 2012 fourth international conference on communication systems and networks (COMSNETS 2012). 2012. IEEE.

21. Manzoor, A., C. Patsakis, J. McCarthy, G. Mullarkey, S. Clarke, V. Cahill, and M. Bouroche. Data sensing and dissemination framework for smart cities. in 2013 International Conference on MOBILe Wireless MiddleWARE, Operating Systems, and Applications. 2013. IEEE.

22. Hoh, B., T. Yan, D. Ganesan, K. Tracton, T. Iwuchukwu, and J.-S. Lee. TruCentive: A gametheoretic incentive platform for trustworthy mobile crowdsourcing parking services. in 2012 15th International IEEE Conference on Intelligent Transportation Systems. 2012. IEEE. 